\title{
Exploring Design Science Research Project Characteristics - An Initial Empirical Investigation
}

\author{
Michael Werner \\ Copenhagen Business School \\ mwe.acc@cbs.dk
}

\begin{abstract}
Design science research (DSR) has gained increased attention in the past decades. This type of research is commonly organized as research projects that need to be managed thoroughly to achieve the intended outcomes. However, very little empirical data are available that provide insights into the nature of DSR projects. This this study aims to better understand DSR projects as a foundation for further studies. It provides first empirical data about DSR project characteristics that have been collected in eight semi-structured interviews with experienced scholars. The data show that DSR projects are heterogeneous in terms of size, complexity, duration, budget and research outputs. They have in common that they focus on relevance, the design of artifacts, evaluation and the involvement of industry partners. A comparison with related meta-studies reveals that research methods employed in DSR projects differ significantly from the ones identified in the meta-studies.
\end{abstract}

\section{Introduction}

Design Science Research (DSR) is a type of research that has gained increased attention in the international scientific community over the past decades [1]. DSR is especially prevalent in German speaking countries [2], [3] where information systems research (ISR) has traditionally been closely related to the natural sciences. It is also common in research communities in Anglo-Saxon countries [4] where it shows a tendency to positivist, behaviouristic research methods [5]. Several scholars have contributed to clarify the role, differences, relationships and interaction of design science, natural science [6], social science [7] and sciences of the artificial [8]. Guidelines have been published on how to conduct DSR in a structured and rigorous manner [3], [9]-[11] and for positioning DSR results in the academic arena [1]. These guidelines are helpful to understand the main components of design science-oriented research and how to structure scientific publications. Scientific activities are commonly carried out as projects, formally or informally. These need to be managed and supervised. Ac- cording to the Project Management Institute [12] projects produce tangible or intangible outputs, are performed by people, constrained by limited resources, temporary, unique and progressive which means that they advance in steps. All these characteristics also apply to research work in general and to DSR in particular. However, very little empirical information has been published yet that provides insights into the nature of DSR projects and how they are actually carried out.

Scholars have pointed out that the sciences of the artificial, natural and social sciences are complementary [7]. Their interaction is important for the progress of ISR [6]. Hence, successful DSR projects that contribute to the accumulation of scientific knowledge are a part of this cycle and an important prerequisite for the progress of ISR in general. Carrying out research projects is a difficult and resource intensive task. The question arises if DSR projects differ significantly compared to other types of projects and how these differences might be important to carry out such projects successfully. This question can hardly be answered substantially without any empirical foundation.

This study aims to provide an empirical contribution to build a foundation for answering this question. It follows the call of Aggerfalk [13] for more empirical contributions related to DSR. It presents and discusses qualitative and quantitative data related to DSR projects that have been collected in eight semi-structured interviews. These interviews were carried out with senior researchers that possess substantial experience in conducting DSR. The interviewed scholars devoted about two thirds of their scientific activities to DSR over an average scientific career of 21 years. The interview partners resided in three different countries on three different continents at the time of interviewing.

The interviews covered a variety of different aspects. This article focusses on the empirical data relating to the characteristics of DSR projects in terms of complexity and duration, funding, research methods and research outputs. It shows that DSR projects are very heterogeneous in terms of size, complexity, duration, and budget as well as research outputs. They are similar in regards to the focus on the design, development or construction of artefacts, the relevance and evaluation of produced research output and the involvement of users and project 
partners from the application domain. The collected data is discussed in detail in the following sections.

The next section describes the background information and refers to related scientific literature. It follows a description of the employed methodology. The empirical results are presented and discussed afterwards. The study closes with a summary and outlook to future research in the last section.

\section{Background}

Of particular interest for this study are scientific publications that deal with DSR projects in ISR. A review of literature related to DSR and research projects ${ }^{1}$ indicated that a substantive amount of articles has been published in the leading information system journals in the past decade that are related to DSR. Approximately half of these publications are related to contributions in regard to the understanding and theoretical foundations of DSR. They deal with DSR on a meta-research level. The other half claim to have applied a DSR research methodology to create the research results that are presented. No publications have been identified that aim to provide empirical data on DSR projects as such. This type of information is extremely rare. Qualitative data can partly be derived from those DSR publications that provide details about the related research project such as described, for example, in [14] or [15]. However, this kind of information is very limited to get an overall understanding about the characteristics of DSR projects. It is used to describe the context in which requirements were identified, the artefacts were developed and evaluated to discuss and interpret the research contribution. The literature review results remain similar even if the investigation is extended to other publication outlets.

A variety of empirical publications deal with DSR research methods and methodologies. Empirical investigations done by [16], [17] or [2], [18], [19] provide insights about the prevalent research methods whereas a variety of publications discuss the structure of DSR [1], [10], [20]. Other publications discuss evaluation aspects [21], [22] or the overall characteristics of DSR as a specific type of research [3], [6], [9], [23]. These publications are helpful to understand the nature of DSR and to carry out such research. However, they provide little insights into how DSR projects look like in practice and what kind of challenges are associated to them.

Research projects are the foundations of scientific activities. Interestingly research related to the nature and

\footnotetext{
${ }^{1}$ A Scopus search for the term 'Design Science' including the AIS Senior Scholars' Basket of Journals yielded 72 hits. 37 of these articles refer to meta-DSR whereas 33 refer to applied DSR-papers.
}

management of research projects in general seems to be rare in the ISR community. ${ }^{2}$ Martin [24] provides empirical data related to IT projects, whereas Lesca and Caron-Fasan [25] explore factors contributing to the failure and abandonment of a specific type of IS projects based on the survey of 39 projects over a 20 year period. These publications refer to commercial projects which raises the question if the derived results can also be applied to scientific projects. vom Brocke and Lippe [26] take a project management perspective on DSR projects. Their research relies on a literature review of design science and project management papers. They identify eight characteristics that distinguish design science-oriented research projects from traditional project types. They conclude that DSR projects differ from commercial projects because they focus on problem-solving and have an unknown outcome. They produce novel but also generally applicable results, combine creative, unpredictable work with regular, pre-defined tasks, have a high percentage of evaluation and testing activities. The measures of success vary between various stakeholders with primarily qualitative success measures, and the success of such projects is not immediately critical to the organizational success. Other publications deal with management and organizational aspects of research projects in DSR. vom Brocke and Lippe [27], [28] published research on management guidelines for collaborative IS research projects. These publications provide information about the challenges of managing DSR projects but they do not provide empirical data.

\section{Methodology}

Empirical data on DSR projects is difficult to gather. Research projects are commonly complex and span over a comparably long time period. Very little information on research project details is publicly available. Information on running and completed research projects can sometimes be found on websites of the involved scientific institutions. However, these sites commonly provide information about the research problem and research outputs. They provide little information about the characteristics and the nature of the underlying research project. They are also frequently taken offline when the project is over. Other data sources are publically accessible national research project databases. Examples are the GEPRIS [29] or the German publicly state funded projects database [30]. A major obstacle to use data from these sources is the uncertainty in regards to the

\footnotetext{
${ }^{2}$ A literature search in Scopus for 'research project' relying on The Web of Science classification for ISR publications did not reveal any scientific literature in respect to research projects in information systems that provide empirical data spanning more than a single research project.
} 
nature of each research project. It remains unclear which project can actually be considered a DSR project and which not.

This study followed a different path by collecting empirical data from those who actually carry out DSR projects. The data that is presented and interpreted in this study was gathered by semi-structured interviews to collect qualitative and quantitative data related to DSR projects. The data collection primarily followed the guidelines published by Myers and Newman [31] who suggest the usage of a dramaturgical model.

The conducted interviews ought to be of exploratory in nature. Taking into account that little empirical information had been available on the matter of investigation, researchers were identified who possessed an extensive experience in DSR with an international background and reputation. Candidates were considered experts if they possessed expert knowledge in DSR through substantial personal experience which exceeds common knowledge. This is usually the case if individuals are in charge of the design, implementation or control of decisions relevant to the research topic [32]. 16 potential interviewees were initially identified, eight agreed to participate. It should be noted that the selection of experienced and internationally renowned researchers created a significant bias on the gathered data. Several scholars suggest to consider a stratified approach by selecting interviewees with different positions in an organization and varying expertise to prevent an elite bias [33]. Given the lack of empirical data on DSR projects in general a focus on selected senior experts was seen as being acceptable for an initial investigation. The inclusion of additional perspectives by interviewing different project stakeholders is foreseen for future investigations. ISR can be seen as an intermediary discipline [5] which is often either located in the business or computer science departments, schools or faculties of a university. To cater for the interdisciplinary nature at least one scholar with a background in business, information systems and computer science was included.

The interviews were semi-structured by using a questionnaire. It included a mix of open and closed questions. Semi-structured interviews were used to be able to receive a standardized set of quantitative data on DSR project characteristics on the one hand but also to gather as much qualitative data as possible on the other. It was therefore preferred over completely structured or unstructured interviews respectively. The interview questionnaire is available in the Appendix.

The interviews were carried out as face-to-face meetings due to potentially sensitive data and confidentiality concerns. They took place during the fourth and 16th of December 2014. The response rate for all items discussed in this study was $95.83 \%$. All interviews were recorded, transcribed and coded for analysis purposes.
Table 1 Interview Partner Details

\begin{tabular}{|c|c|c|c|}
\hline $\begin{array}{c}\text { Inter- } \\
\text { viewee }\end{array}$ & $\begin{array}{c}\text { Scientific } \\
\text { Background }\end{array}$ & Country & $\begin{array}{c}\text { Research } \\
\text { Experience }\end{array}$ \\
\hline 1 & IS & USA & 35 \\
\hline 2 & IS & AUS & 21 \\
\hline 3 & Business & USA & 18 \\
\hline 4 & IS & USA & 15 \\
\hline 5 & IS & GER & 17 \\
\hline 6 & IS & AUS & 30 \\
\hline 7 & CS & AUS & 21 \\
\hline 8 & CS & AUS & 13 \\
\hline
\end{tabular}

All interviewees were professors at universities and internationally renowned scholars with an average research experience of 21.25 years. Table 1 provides a general overview of the participating researchers. The interviews covering the questions described in this study took 33.14 minutes on average.

\section{DSR Project Characteristics}

\subsection{Distinguishing Characteristics}

The first question served to receive information about the interviewees' understanding and definition of DSR. The interview partners provided answers of differing length and depths to this question. 24 aspects were identified on the basis of the provided answers that characterize DSR.

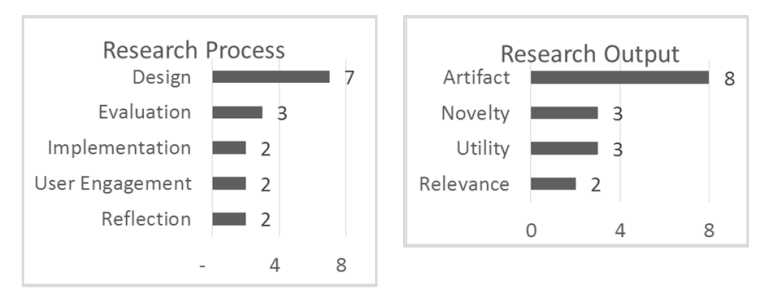

Figure 1 Distinguishing Characteristics

Figure 1 shows those aspects that were mentioned by at least two interviewed scholars. These relate to the research process and research output. The most commonly mentioned distinguishing characteristic was the design (7) of an artefact (8) such as methods, algorithms, sociotechnical artefacts, programs, designs and processes. Three of the interviewees directly mentioned that 'The design of the artefact distinguishes DSR from any other research'. Two interviewees stressed also the importance of the coding and implementation or production of an artefact. Other important characteristics that were mentioned refer to user engagement (2), evaluation (3) of and reflection (2) on the research output. Several interview partners stressed the need to create new artefacts or knowledge (3), relevance of the research output (2) and its utility (3), for example, in terms of improved performance or quality. Some scholars pointed 
out that DSR projects commonly involve larger research resources than traditional empirical research, that those projects commonly exhibit a higher complexity and require different skill sets of the involved researchers.

\subsection{Expertise and Responsibilities}

Questions 2 to 4 were supposed to investigate the specific experience of the interview partners and their roles in DSR projects. The responses showed that the interviewed scholars devoted on average $61 \%$ of their research effort on DSR. Some scholars had participated in many DSR projects (maximum 40) whereas some researchers participated in just a few (minimum 2). On average the interview partners had participated in 9.5 DSR projects. There was no pattern identifiable between information systems and computer science scholars in terms of project involvement. The one business scholar was involved in fewest projects compared to the other interviewed scholars. This complies with his perception that DSR projects are comparatively rare in his scientific discipline.

On average the interviewees named approximately three different roles of project involvement. All but one of the interview partners were engaged in DSR projects as researchers or developers on an operational level during their own PhD studies and early in their academic careers. Six were engaged with management responsibilities as project managers, project leaders, sub-project managers, or scientific project coordinators. Three mentioned that they were responsible for supervising $\mathrm{PhD}$ students or acting as a senior academic advisor for more junior colleagues during these projects. Three researchers were commonly involved as project designers or initiators without actually carrying out or managing the project itself. Two also mentioned their role as an intermediary or mediator between project partners and researchers. One researcher mentioned his consulting role in DSR projects that were mainly carried out by projects partners from industry.

\subsection{Project Complexity and Duration}

Questions 5 to 7 were designed to investigate the size of DSR projects in terms of involved researchers and other project stakeholders as well as the overall duration of such projects. On average a DSR project involved four to five project group members. The smallest mentioned DSR project involved just the interviewed researcher alone. Some scholars also mentioned much larger research groups. The largest mentioned project involved several dozens of researchers from different universities. Three interviewees provided additional in- formation on the type of involved researchers. According to their information DSR projects are commonly staffed with at least one senior academic and one or more $\mathrm{PhD}$ or master students. Two scholars mentioned that staff employed by industry project partners commonly also formed part of the project team although this constellation seemed to refer to larger projects.

On average three different project partners were involved in a single DSR project. Typically project partners were companies, public organizations such as government agencies or the public administration and nonfor-profit organizations. Other research institutions like universities were mentioned by the majority of the interviewees as a typical research partner. All interviewed scholars concluded that at least one project partner from the industry or the public sector - that represented the application domain participated in a DSR project.

In terms of duration a DSR project lasted three to four years on average. The longest mentioned project lasted about ten years whereas the shortest micro project was finished after just three months. Several researchers pointed out that it is very difficult to actually determine the start and end of a project. One scholar noted that the publication process commonly continued even after the project itself had ended.

In summary the provided answers revealed that a standard DSR project at least included three researchers. One junior researcher was usually responsible for the analysis, design and evaluation, a second junior researcher was responsible for the implementation and a senior academic provided advice, supervision and managed the project. A DSR project always required at least one project partner representing the application domain. It was most commonly a project partner from industry. Several interviewees pointed out that the involvement of a project partner is a critical prerequisite for a DSR project. It is generally hard to define when such a project actually starts and ends, but typical projects lasted three to four years although the publication process could take much longer. They involved four to five researchers and three different project stakeholders. These characteristics refer to standard DSR projects. In large DSR project many researchers worked together, these projects could involve up to ten project partners and could span over a decade or longer.

\subsection{Project Funding}

Questions 8 and 9 related to the extent and sources of funding to carry out DSR projects. These questions were delicate due to the potential confidentiality of the provided information. The received answers varied significantly and most of the interview partners pointed out that their figures were only rough estimates and just provided a broad overview. Three of the interviewees did 
not provide any figures. One interview partner stated that no external funding was available for any of the conducted DSR projects. These were exclusively funded internally by the university. The only support were minor travel costs reimbursements and non-monetary support from project partners. One scholar noted that the biggest DSR-related project in which he was involved in was set up as a collaborative project and funded with approximately 41 million USD. This project included the setup of a whole research centre and can probably be seen as unique. Another scholar stated that the largest conducted DSR project was externally funded with approximately 6 million USD. One scholar stated the maximal budget with approximately 1.2 million USD and one further researcher 250 thousand USD. The average calculated budget based on the received responses was 261 thousand USD.

The figures become more insightful when taking provided qualitative information into account. One scholar pointed out that a typical research project that ran over three years was usually funded with 250 to 500 thousand USD. This observation was supported by a second scholar calculating the costs for a typical DSR project that involved two paid researchers, one responsible for the design, the other for implementation, of approximately 125 thousand USD a year.

In terms of funding sources six interviewees mentioned more than one primary source of funding. Six stated that public grants from government or similar institutions like the European Union were one primary source. Four stated industry partners or industry associations as a primary source. Five referred to university internal funding as a major fund giver.

\subsection{Research Methods}

Several meta-studies have analysed the research methods employed and described in ISR publications. The objective by asking question 10 was to collect information about the actual usage of research methods in DSR projects to compare if those described match those that have been identified in the literature.

The provided answers were partly difficult to summarize because the understanding and differentiation between research methods, methodologies and paradigms are inhomogeneous in ISR itself [34]. The different academic background of the interviewed scholars might also have contributed to the heterogeneity of received answers. To harmonize the received information the provided answers were grouped into categories which were derived from already existing studies [2], [16]-[19].

Figure 2 summarizes the received answers. It shows the distribution of each mentioned method. The interviewers on average mentioned four to five different research methods that they typically employ in DSR projects. The development of an artefact through prototyping or other types of programming was mentioned most frequently (7) followed by survey as the second most common research method (4).

Figure 3 and Figure 4 show the results compared to the data received from other studies. Palvia et al. [16], [17] conducted a literature review to capture the trends in research being conducted in the Management Information Systems (MIS) field covering seven leading MIS journals over the period of 1993 to 2003. Wilde and Hess [2], [18] as well as Schreiner et al. [19] investigated the methodological profile of publications in the BISE journal. The results of these studies are particularly interesting because the studies presented by Palvia et al. [16], [17] provide an overview of journals representing mostly publications originating from an AngloSaxon research community whereas the BISE journal is considered to represent especially design-science oriented research from continental Europe. Both sets of studies use different terms and categories to classify research methods. This studies relies on the classification used by Schreiner et al. [19] as this publication actually incorporate the publications from Palvia et al. [16], [17] and extents the ones by Wilde and Hess [2], [18]. ${ }^{3}$ The research methods identified in the study at hand as listed in Figure 2 where grouped accordingly ${ }^{4}$ for representation in Figure 3 and Figure 4.

The initial assumption would be that the methodological profile identified by this study should be similar to the one presented by Schreiner et al. [19] because the latter one is assumed to represent especially design science-oriented research.

\footnotetext{
${ }^{3}$ The classifications are shown in Figure 3. Argument based deduction includes library research and literature analysis, case study includes field study, quantitative-empirical includes survey and secondary data, conceptual deduction includes frameworks and conceptual model, formal deduction includes mathematical model, qualitative-empirical includes qualitative research and interview.
}

\footnotetext{
${ }^{4}$ Quantitative - empirical includes surveys, data analysis and eye-tracking, qualitative - empirical includes interviews, observations, focus groups, thinking aloud and video analysis, cases studies includes field studies, formal deduction includes complexity measuring and algorithm development, prototyping includes system development, action research includes action design research.
} 


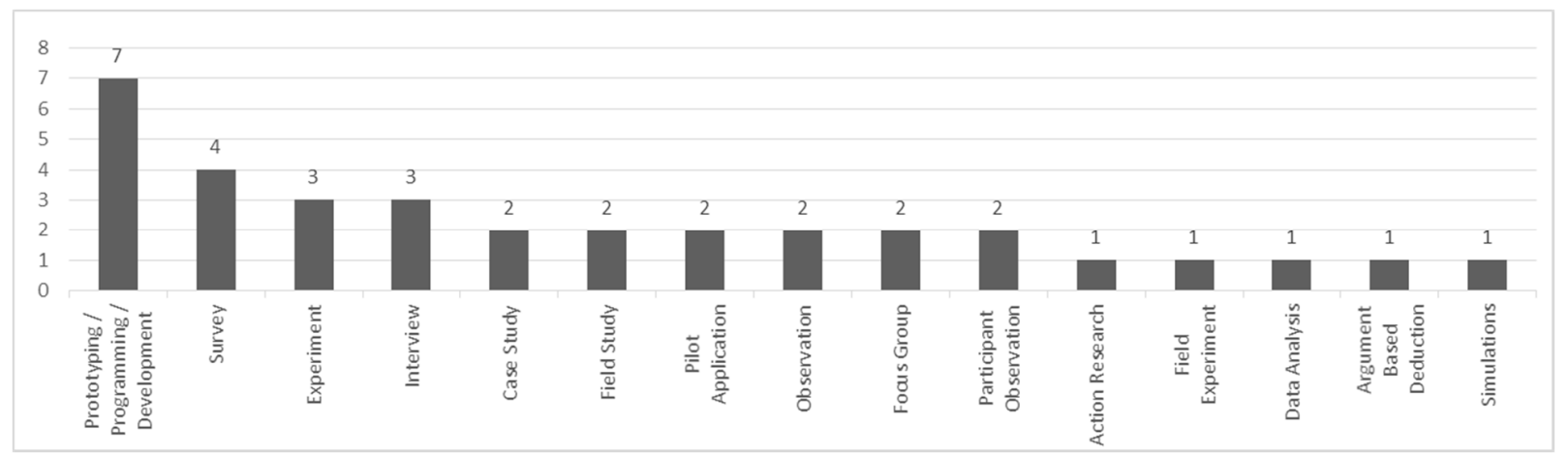

Figure 2 Research Methods Used in DSR Projects

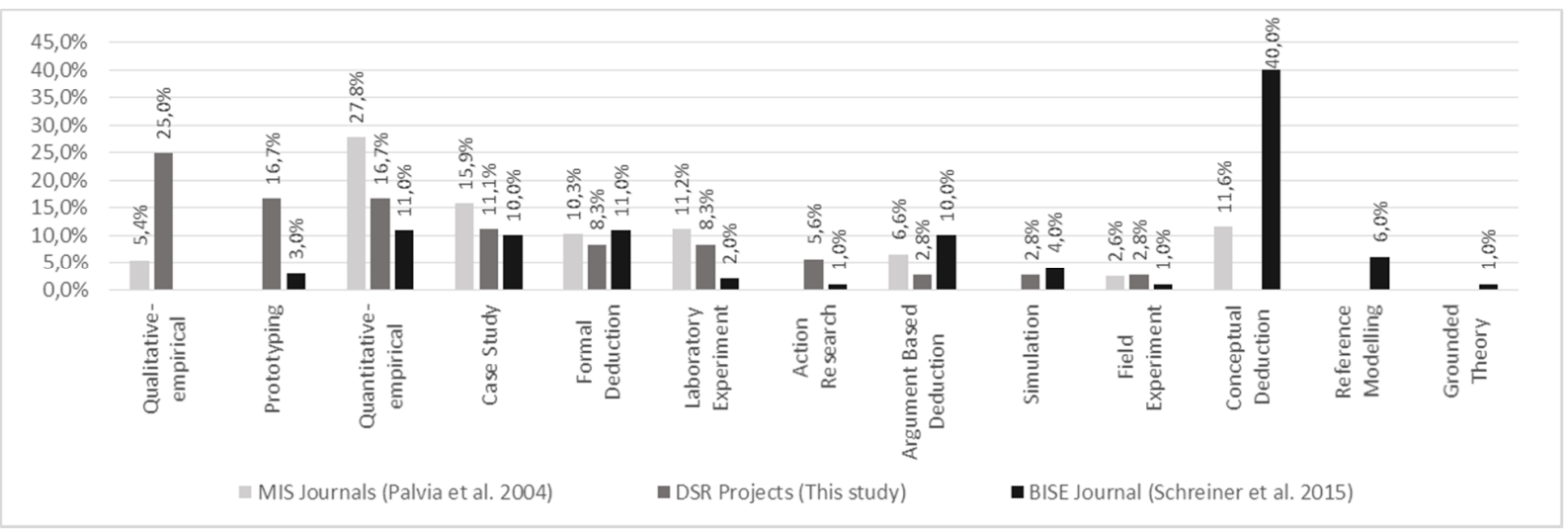

Figure 3 Research Methods in Information Systems Publications and DSR Projects

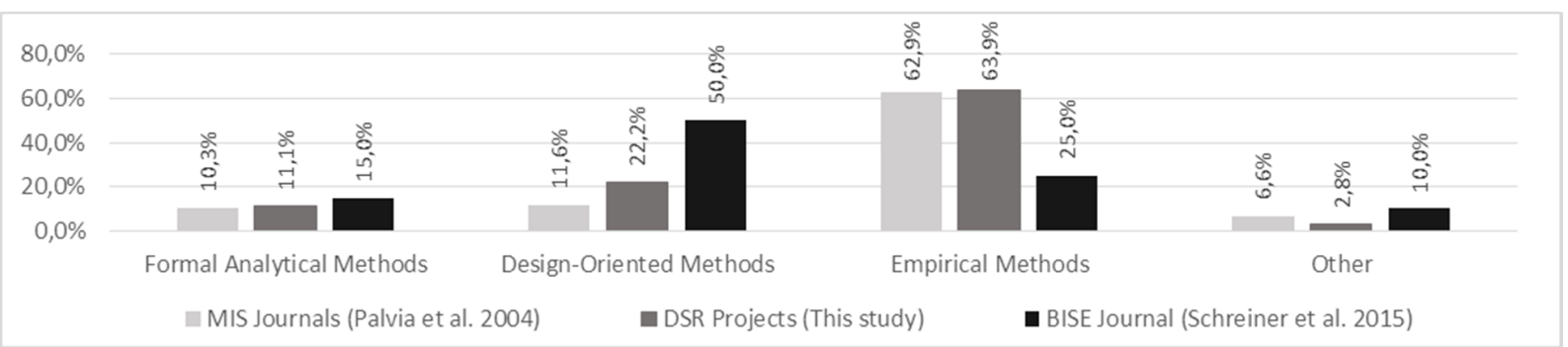

Figure 4 Methodological Profile in Information Systems Publications and DSR Projects

Figure 3 shows that this was not the case. The most typical research method identified by Schreiner et al. [19] was conceptual deduction, a research method that uses semi-formal models for logical deduction to create the research output. Research methods that would fall under this category as, for example, conceptual modelling were not mentioned by any of the interview partners during the conducted interviews. The same is the case for reference modelling.

Figure 4 provides a more comprehensive overview. The different research methods were grouped into four different categories following the categorization introduced by Schreiner et al. [19]. It actually shows that the profile of research methods identified in this study is more similar to the MIS profile than the BISE profile as both studies show the predominance of empirical research methods. On the other hand the percentage of design-oriented research methods identified in this study is double as high as the usage identified by Palvia et al. [16], [17].

There are several possible explanations for this observation. First, both sets of studies, [16], [17] as well as [2], [18] and [19], focus on the primary research method that was employed. The answers given by the interview partners in the study at hand revealed that different research methods were used in a DSR project in different phases. Schreiner et al. [19] also investigated the usage of secondary research methods. Within this data set the 
usage of empirical research methods rose to $37.5 \%$. Second, it can be assumed that the [2], [18] and [19] studies represent the research results from German speaking countries whereas the study at hand incorporates DSR researchers with an international background. Third, it should be accounted for that the answers received from the different scholars did not provide concrete information of how often these research methods were employed. This information was derived indirectly by referring to the frequency of nominations. Fourth, $37.25 \%$ of the interviewed scholars stated that evaluation of the research results was an important aspect of DSR. This commonly required the application of an empirical research method. Over two thirds of the research methods suggested for evaluation purposes by Venable et al. [21], for example, can be classified as empirical research methods. It can be assumed that the need for rigorous evaluation required the application especially of empirical research methods in the evaluation phase of DSR projects leading to a high proportion of empirical methods that were used in such projects.

In summary the results show that the most common cited research method were prototyping as a classical design-oriented method but that the empirical research methods made up almost two thirds of the employed research methods compared to $22 \%$ of design-oriented research methods overall. The identified profile lies between the profiles identified by the studies carried out by Palvia et al. [16], [17] and Schreiner et al. [19]. Although there are different explanations for some of the observed variation between the studies it still remains unclear why certain types of research methods do not show up as expected in the publications that are a result of these projects. This is especially the case for qualitative-empirical research methods and prototyping. Several interview partners provided anecdotal evidence about challenges in publishing DSR results. Five interviewees reported that publishing DSR research is extremely difficult. They stressed the need for reviewers to get familiar with the concept of DSR and mentioned that editors and reviewers often miss the knowledge how DSR papers should look like. In contrast another scholar emphasized that the publications process is exactly the same for DSR output, but that the success depends on the quality of the research and manuscript as it is the case for any type of research.

A reasonable explanation for the observation that several research methods seem to be underrepresented in the scientific publications might be a lack of familiarity of editors and reviewers with specific design science-oriented research methods. It could also be the result of conscious or unconscious choices made by the authors to focus on those research methods they feel will be most successful to get published. The consequence might be a higher representation of traditional quantitative empirical research methods that editors and reviewers are more accustomed with. But this explanation can neither be confirmed nor rejected by the gathered data. Future research should look into this matter more closely to identify if a discrimination of particular research methods in terms of publication indeed takes place and what the consequences for the progress of ISR could be.

\subsection{Academic Research Outputs}

Questions 11 and 12 were used to receive information about the academic output produced in DSR projects. The received answers revealed various interpretations of the term artefact. In general a particular DSR project focused on one primary artefact. This could be accompanied by different other related artefacts such as models that were used to create a prototype or the methods to use it. Depending on the size of a project each artefact could consist of different sub-artefacts.

The numbers related to the produced scientific publications varied. The difficulty to provide concrete numbers also referred to the uncertainty discussed in question 7 with regard to the actual duration of a research project. On average DSR projects commonly lead to three or four publications. Some scholars pointed out that the scientific output usually did not solely consist of publications about the created artefacts which were being written during the active project but also meta-publications about DSR that dealt with the insights that had been gained throughout the research projects. Such publications were sometimes produced a considerable time span after the project itself had ended.

\subsection{Project Categories}

The answers discussed in the previous sub-sections show that DSR projects vary significantly in terms of duration and complexity, funding sources and amount of research outputs. DSR projects can be very small or extremely large. The collected data shows that DSR projects can be categorized as small, medium or large.

Table 2 provides an overview of the characteristics for the different project categories based on this study's findings.

\begin{tabular}{|l|c|c|c|}
\hline $\begin{array}{c}\text { Characte- } \\
\text { ristic }\end{array}$ & Small & Medium & Large \\
\hline $\begin{array}{l}\text { Research } \\
\text { Group Size }\end{array}$ & $1-2$ & $3-6$ & $>6$ \\
\hline $\begin{array}{l}\text { Project } \\
\text { Partners }\end{array}$ & 1 & $2-4$ & $>4$ \\
\hline $\begin{array}{l}\text { Project } \\
\text { Duration }\end{array}$ & $<3$ years & $3-4$ years & $>4$ years \\
\hline
\end{tabular}




\begin{tabular}{|l|c|c|c|}
\hline $\begin{array}{l}\text { Project } \\
\text { Budget }\end{array}$ & $\begin{array}{c}< \\
\$ 250,000\end{array}$ & $\begin{array}{c}\$ 250,000- \\
\$ 500,000\end{array}$ & $>\$ 500,000$ \\
\hline $\begin{array}{l}\text { Project } \\
\text { Funding }\end{array}$ & UI & PG, IP, UI & PG, IP, UI \\
\hline $\begin{array}{l}\text { Publica- } \\
\text { tions }\end{array}$ & $1-3$ & $3-6$ & $>6$ \\
\hline
\end{tabular}

Table 2 DSR Project Categories

\section{Conclusion}

DSR is an important type of research in information systems research. It has gained increased attention in different research communities around the world and on an international level. Research has been published to clarify the role, differences, relationships and interaction of design science research with other sciences, to provide guidelines on how to conduct DSR and for publishing DSR results. However, these publications provide little empirical evidence about the way DSR is actually carried out in real research projects.

This study contributes to the body of knowledge by presenting first empirical evidence about DSR projects characteristics. These have been identified through the analysis of empirical quantitative and qualitative data that was collected in semi-structured interviews with eight DSR-experienced senior scholars. All DSR projects have in common that they deal with the design, development and construction of artefacts that have to be novel, relevant and useful. The evaluation of such artefacts is an important part in DSR projects as well as their implementation and reflection upon. User engagement is another crucial characteristic. Almost all DSR projects rely on the cooperation with at least one project partner from industry or a public organization that represents the application domain. Besides being involved in the actual research activities scholars fill out a variety of different roles in DSR projects as managers, academic advisors and project designers. They also act as intermediaries between science and practice or as consultants for project partners.

A DSR project usually focusses on the design of a primary artefact which can consist of sub-artefacts or which can be accompanied by additional artefacts such as instantiations and methods for its application.

DSR projects differ significantly in terms of complexity and duration, funding, and outputs. Three to six researchers work in a typical medium-sized DSR project. Such a project lasts three to four years starting and ending with the official project funding. This period is longer if the project initiation and the complete publication process is taken into account. Two to four different external project partners work together in a typical medium-sized DSR project. These projects are usually funded by public grants, industry partners, internally or as a mix of all three. The budget lies between 250,000 and 500,000 USD. The scientific output varies but a typical medium-sized DSR project produces three to six different publications.

A variety of research methods is used in DSR projects. The most prominent is prototyping. Overall design-oriented research methods just account for $22 \%$ of the employed research methods whereas $64 \%$ account for empirical research methods and $11 \%$ for formal analytical ones. The analysis of the identified profile reveals an anomaly. The profile differs from those identified by scholars for DSR-oriented journals and those for MIS journals. It is located between those two profiles. The high percentage of empirical research methods can partially be explained by the need to apply different research methods within a single project where especially the evaluation primarily requires empirical research methods. However, it does not satisfactorily explain why certain research methods are underrepresented which is especially the case for qualitative-empirical research methods and prototyping. Anecdotal evidence from the interviewed scholars suggests that editors and reviewers are not yet as familiar with DSR research as with other types of research. As a consequence DSR studies relying on typical design science-oriented research methods such as prototyping might not get published as expected. The deviation could also be the result of conscious or unconscious choices made by the authors of DSR studies to focus on those research methods they feel will be most successful to get published. The data gathered during this study can neither confirm nor reject these hypotheses. Future research should look into this matter more closely to identify if a discrimination of particular research methods indeed takes place and what the consequences for the progress of ISR could be.

The gathered empirical data show that DSR projects are complex and multifaceted phenomena. So far research on managing DSR projects is extremely scarce. Guidelines do exist that support academics in carrying out different research activities and that provide an overview of the DSR research process. There is also a rich body of knowledge on project management in general and the management of IT or software development projects in particular. However, little research is concerned about how this knowledge can be applied to support researchers to organize and manage DSR research projects including the project initiation and setup, supervision of junior researchers, communication with project stake-

\footnotetext{
${ }^{5}$ Project funding sources: $\mathrm{UI}=$ University internal, $\mathrm{PG}=$ Public Grants, IP = Industry Partner
} 
holders and the evaluation of the research output in realistic scenarios to be able to assess the utility of the produced artefacts. These activities are challenging and different to those usually associated with in the context of academic responsibilities. Future research should investigate how researchers can be supported in this respect.

A major limitation of this study is the relatively small number of scholars that have been interviewed. These scholars are internationally renowned in their field of expertise and it can be assumed that the gathered information provides a reliable overview. However, additional quantitative empirical research is necessary to further substantiate the conclusions drawn in this paper. This is planned for future research. The focus on senior scholars also introduced a specific bias on the gathered data as potential important perspectives from other project stakeholders, such as junior researchers or project partner representatives, were neglected. Further interviews with diverse stakeholders are planned in order to assess if such a bias affects the presented data. Future research could also deal with the applicability of established management standards for DSR projects based on the presented findings.

\section{Acknowledgement}

The described research would not have been possible without the kind support of the different interview partners that devoted their time and best knowledge to provide meaningful answers. I would like to thank Shirley Gregor, Boualem Benatallah, Tilo Böhmann, Roger S. Debreceny, Alan. R. Hevner, Fethi Rabhi and the other researchers who participated as interview partners in this study as well as Markus Nüttgens for his ongoing encouragement and sponsorship.

\section{References}

[1] S. Gregor and A. R. Hevner, "Positioning and Presenting Design Science Research for Maximum Impact," MIS Quarterly, vol. 37, no. 2, pp. 337-355, 2013.

[2] T. Wilde and T. Hess, "Forschungsmethoden der Wirtschaftsinformatik," Wirtschaftsinformatik, vol. 49, no. 4, pp. 280-287, 2007.

[3] H. Österle et al., "Memorandum on design-oriented information systems research," European Journal of Information Systems, vol. 20, no. 1, pp. 7-10, 2010.

[4] R. Baskerville, K. Lyytinen, V. Sambamurthy, and D. Straub, "A Response to the Design-oriented Information Systems Research Memorandum," European Journal of Information Systems, vol. 20, no. 1, pp. 11-15, 2010.
[5] C. Schauer, Die Wirtschaftsinformatik im internationalen Wettbewerb. Wiesbaden: Gabler, 2011.

[6] S. T. March and G. F. Smith, "Design and natural science research on information technology," Decision support systems, vol. 15, no. 4, pp. 251-266, 1995.

[7] S. Gregor and R. Baskerville, "The Fusion of Design Science and Social Science Research," ISF 2012, 2012.

[8] H. A. Simon, The Sciences of the Artificial. Cambridge, Mass.: MIT Press, 1996.

[9] A. R. Hevner, S. T. March, J. Park, and S. Ram, "Design Science in Information Systems Research," MIS Quarterly, vol. 28, no. 1, pp. 75105, Mar. 2004.

[10] K. Peffers, T. Tuunanen, M. Rothenberger, and S. Chatterjee, "A Design Science Research Methodology for Information Systems Research," J. Manage. Inf. Syst., vol. 24, no. 3, pp. 45-77, 2007.

[11] A. Hevner and S. Chatterjee, "Design Science Research in Information Systems," in Design Research in Information Systems, vol. 22, Boston, MA: Springer US, 2010, pp. 9-22.

[12] Project Management Institute, Ed., A guide to the Project Management Body of Knowledge (PMBOK guide), sixth edition, Sixth edition. Newtown Square, PA: Project Management Institute, 2017.

[13] P. J. Ågerfalk, "Insufficient theoretical contribution: a conclusive rationale for rejection?," European Journal of Information Systems, vol. 23, no. 6, pp. 593-599, Nov. 2014.

[14] B. Kuechler and V. Vaishnavi, "On Theory Development in Design Science Research: Anatomy of a Research Project," European Journal of Information Systems, vol. 17, no. 5, pp. 489504, Oct. 2008.

[15] J. Weedman, "Client as designer in collaborative design science research projects: what does social science design theory tell us?," European Journal of Information Systems, vol. 17, no. 5, pp. 476-488, Oct. 2008.

[16] P. Palvia, E. Mao, A. F. Salam, and K. S. Soliman, "Management Information System Research: What's There in a Methodology?," Communications of the Association for Information Systems, vol. 11, pp. 289-309, 2003.

[17] P. Palvia, D. Leary, E. Mao, V. Midha, P. Pinjani, and A. F. Salam, "Research Methodologies in MIS: An Update," Communications of the Association for Information Systems, vol. 14, pp. 526-542, 2004. 
[18] T. Wilde and T. Hess, "Methodenspektrum der Wirtschaftsinformatik: Überblick und Portfoliobildung," Arbeitspapiere des Instituts für Wirtschaftsinformatik und Neue Medien, LMU München, vol. 2, 2006.

[19] M. Schreiner, T. Hess, and A. Benlian, "Gestaltungsorientierter Kern oder Tendenz zur Empirie? Zur neueren methodischen Entwicklung der Wirtschaftsinformatik," Arbeitsbericht, LMU München, 2015.

[20] J. Venable, "A Framework for Design Science Research Activities," Information Resource Management Association Conference, Washington, DC, USA, 2006.

[21] J. Venable, J. Pries-Heje, and R. Baskerville, “A Comprehensive Framework for Evaluation in Design Science Research," Design Science Research in Information Systems. Advances in Theory and Practice, pp. 423-438, 2012.

[22] C. Riege, J. Saat, and T. Bucher, "Systematisierung von Evaluationsmethoden in der gestaltungsorientierten Wirtschaftsinformatik," Wissenschaftstheorie und gestaltungsorientierte Wirtschaftsinformatik, pp. 69-86, 2009.

[23] R. Baskerville, "What design science is not," European Journal of Information Systems, vol. 17, no. 5 , pp. 441-443, Oct. 2008.

[24] A. Martin, "What drives the configuration of information technology projects? Exploratory research in 10 organizations," Journal of Information Technology, vol. 18, no. 1, pp. 1-15, Mar. 2003.

[25] N. Lesca and M.-L. Caron-Fasan, "Strategic scanning project failure and abandonment factors: lessons learned," European Journal of Information Systems, vol. 17, no. 4, pp. 371-386, 2008.

[26] J. vom Brocke and S. Lippe, "Taking a Project Management Perspective on Design Science Research," in Global Perspectives on Design Science Research, Springer, 2010, pp. 31-44.

[27] J. vom Brocke and S. Lippe, "Towards Management Guidelines for Collaborative Research Projects on Information Systems - Learning from Project Management Contingency Theory," in Management of the Interconnected World, A. D' Atri, M. De Marco, A. M. Braccini, and F. Cabiddu, Eds. Heidelberg: Physica-Verlag HD, 2010, pp. 527-534.

[28] J. vom Brocke and S. Lippe, "Identifying and Managing Creative Tasks in Collaborative IS Research Projects: Identifying and Managing Creative Tasks in Collaborative IS Research Projects," Project Management Journal, vol. 44, no. 6, pp. 94-113, Dec. 2013.
[29] Deutsche Forschungsgemeinschaft, "DFG GEPRIS," 2015. [Online]. Available: http://gepris.dfg.de/gepris/.

[30] Deutsche Bundesregierung, "Förderkatalog," 2015. [Online]. Available: http://foerderportal.bund.de/foekat/jsp/StartAction.do.

[31] M. D. Myers and M. Newman, "The Qualitative Interview in IS Research: Examining the Craft," Information and Organization, vol. 17, no. 1, pp. 2-26, Jan. 2007

[32] M. Pfadenhauer, "Das Experteninterview," in Qualitative Marktforschung, Springer, 2007, pp. 449-461.

[33] M. B. Miles and A. M. Huberman, Qualitative data analysis: an expanded sourcebook, $2 \mathrm{nd} \mathrm{ed}$. Thousand Oaks: Sage Publications, 1994.

[34] J. Mingers, "Combining IS Research Methods: Towards a Pluralist Methodology," Information Systems Research, vol. 12, no. 3, pp. 240-259, 2001.

\section{Appendix Interview Questionnaire}

1) How would you classify design science research? What distinguishes design science research from other research approaches?

2) How much of your research work do you devote to design science-oriented research (share of research workload dedicated to DSR projects in percentage)?

3) How many design science-oriented research projects did you carry out in your academic career?

4) What role did you play in these projects?

5) How many researchers participated in these projects on average (minimum, maximum)?

6) How many project partners participated in these projects on average (minimum, maximum)? Please also provide information on the type of project partners (private or public sector entities, research institutions etc.)

7) How long did these projects last on average (minimum, maximum)?

8) What was the average (minimum, maximum) size of the projects in terms of budget? Please refer to the overall budget including the budgets for all projects partners if applicable.

9) What were the primary sources of research funding of your DSR projects?

10) What kind of research methods (surveys, experiments, prototyping etc.) did you primarily use to carry out the research tasks?

11) How many different research artefacts were developed during your DSR projects on average (minimum, maximum)?

12) Can you estimate how many scientific publications were created during the projects on average (minimum, maximum) 